\title{
Size and distance perception of the physiognomic stimulus "taketa"
}

\author{
MARTIN S. LINDAUER \\ State University of New York, College at Brockport, Brockport, New York
}

\begin{abstract}
The evocative power of the unfamiliar but expressive stimulus "taketa," considered to be a prototypical example of physiognomy, led to the perception of its enhanced size (Experiment 1) and increased distance (Experiment 2). Comparisons were made of taketa and three other unfamiliar stimuli, one of which was also physiognomic ("maluma"), and six familiar and meaningful shapes. In Experiment $1(N=55)$, a size-matching study was conducted; comparison stimuli were lines of different sizes. The aggressive-seeming taketa was seen as larger than the peaceful-seeming maluma, the two other unfamiliar stimuli, and at least one other meaningful shape. In Experiment $2(N=21)$, predictions about the apparent distance of a large-appearing taketa were supported. A card on which taketa was printed was set farther away, on both ascending and descending trials, than several other stimuli. The results were interpreted as favoring a perceptual basis for physiognomy.
\end{abstract}

Physiognomy refers to the spontaneously seen expressiveness in faces and body gestures, as well as in objects and events (e.g., the sadness of a look, a tree, or a ceremony) (Lindauer, 1984b; Schlesinger, 1980). These evocations involve more than learned associations, since physiognomic effects occur for meaningless, unfamiliar, and irrelevantly paired words and patterns (e.g., a jagged line is angry, red, and Tuesday) (Leijonhielm, 1967; Lindauer, 1984b; Lyman, 1979).

Whether physiognomic stimuli are seen as different from nonphysiognomic stimuli has received less attention than have demonstrations of physiognomy's unexpected presence. An exception is the research of Comalli (1960), who showed that real and apparent (autokinetic) movement differed for physiognomically suggestive objects. Lindauer (1986) also found that the saturation and brightness values of the physiognomic stimulus "taketa" differed from those of another physiognomic stimulus, "'maluma," as well as from two neutral shapes and from some meaningful figures (e.g., an eight-sector circle).

Determining whether the perceived size and distance of the physiognomic taketa would also be affected was the goal of the two experiments reported in this paper. In Experiment 1, it was predicted that taketa, a phenomenologically assertive-appearing stimulus, would be seen as larger than it physically is, and that the sizes of the physiognomically contrasting maluma (a peacefulseeming stimulus) and those of physiognomically neutral shapes should not be enhanced.

A summary of portions of Experiment 1 on size was presented at the 26th Annual Meeting of the Psychonomic Society, Boston, MA, November 22-24, 1985. A briefer version of Experiment 2 on distance was presented at the 56th Annual Meeting of the Eastern Psychological Association, Boston, MA, March 21-24, 1985. The coauthor on that paper was Terrence F. Tully, to whom I am indebted for his fresh ideas and hard work. Address correspondence to Martin S. Lindauer, Psychology Department, State University of New York, College at Brockport, Brockport, NY 14420.
If taketa is perceived to be larger than it is, then its apparent distance should also be affected. The nature of this difference is spelled out in Experiment 2.

\section{EXPERIMENT 1}

\section{Physiognomic Size Perception}

\section{Method}

Stimuli. Nine stimuli, previously used in a color-matching task (Lindauer, 1986), were again selected for this study (see Figure 1). The physiognomic status of maluma and taketa (items 1 and 2, Figure 1) had been defined by consensual validation; that is, these stimuli are frequently referred to and used as prototypical examples by both theorists (e.g., Kohler, 1938/1947) and researchers (e.g., Lyman, 1979). The two meaningless stimuli (items 9 and 10a, Figure 1) were comparable to maluma and taketa in unfamiliarity but not in physiognomy. Five familiar stimuli from the original list (items $3,5,6,7$, and 8 , Figure 1) were included as fillers. ${ }^{1}$ Other details on the selection of the stimuli are found in Lindauer (1986).

The four critical and five filler shapes (the standard stimuli) were matched with lines (the comparison stimuli) that were equal in size to one another $(6.80 \mathrm{~cm})$. Each of the nine standard stimuli was placed at the top of an $8 \times 11$ in. page of an 11-page booklet. Eleven comparison lines were located on the bottom of the page headed by the standard stimulus. For each standard stimulus, there were equal numbers of larger and smaller comparison stimuli; one stimulus was equal in size. The sizes of the comparison stimuli varied in increments that were $2 \%$ of the standard's size. A table of random numbers was used to arrange the orders for each of the comparison stimuli. (The order of comparison stimuli differed for each standard stimulus, but remained the same for that particular standard stimulus.) The pages of each booklet (except for the first sample page) were shuffled. A sample page, containing a training stimulus, preceded the nine sets of stimuli.

Subjects. Fifty-five volunteers ( 13 men and 42 women) from several undergraduate general psychology classes participated for credit. Subjects were run individually.

Instructions. The instructions, printed on the first page of the booklet, were also read aloud by the experimenter. The subjects were told that they were participating in a size-perception task; a sample on the first page was used for illustration. Size was defined as vertical height (i.e., the distance from the top of the stimulus to its bottom). Subjects were instructed to ignore other features (e.g., design variations, width). They were informed that the heights of the comparison stimuli did in fact differ, and that one of them was indeed equal to the height of the 

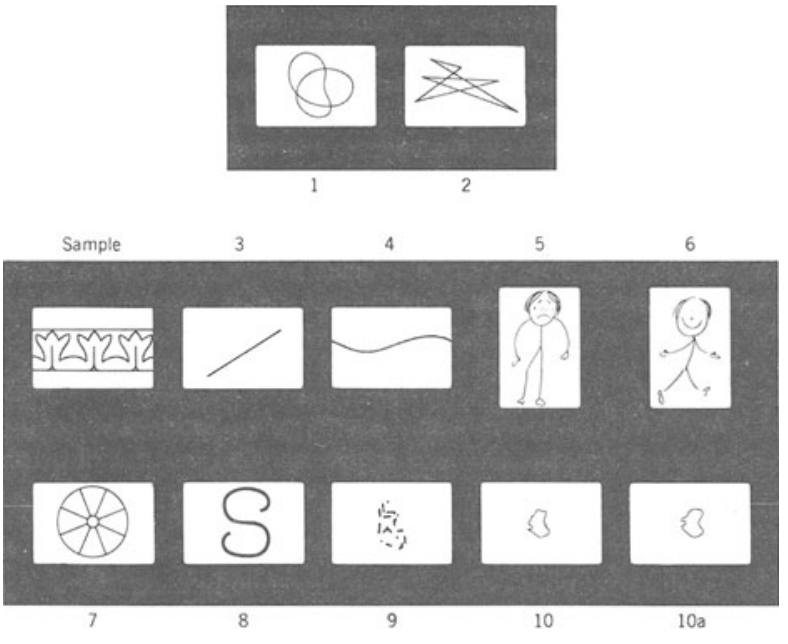

Figure 1. The stimuli used. The physiognomic stimuli are maluma (1) and taketa (2); their comparable unfamiliar stimuli are the nonsense dots (9) and shape (10a). (Item 10 was used in an earlier experiment.) From "Perceiving, Imaging, and Preferring Physiognomic Stimuli” by M. S. Lindauer, 1986, American Journal of Psychology, 99, 236. Reprinted with permission.

standard stimulus. The subjects were told to use the naked eye and not to rely on any aid, such as their fingers or a pencil, to measure the height of the stimuli. They were instructed to work fairly quickly, but at their own pace; there was no time limit. Each page had to be completed before the next one was examined; once a page was turned, the subject could not return to it. Up to $30 \mathrm{~min}$ was required by some subjects, although the times varied.

\section{Results}

Taketa's size, as predicted, was overestimated more than the size of any other stimulus $(M=6.96 \mathrm{~cm})$. The estimation of taketa's size significantly differed $(p<.05)$ from those of the other three critical stimuli (Figure 2; see the darkened areas), including maluma $(M=6.80)$ and both meaningless stimuli, the dots $(M=6.69)$ and the shape $(M=6.67)[F(8,424)=4.07, p<.01] ; t$ tests were used to make comparisons.

Taketa was also perceived as being larger than several familiar stimuli (Figure 2, the white areas). The difference, however, was significant only in the case of the eightsectored circle (item $7 ; M=6.70$ ). In addition, maluma was perceived as being larger than the two physiognomically neutral stimuli, although the differences were not significant $(p>.05)$. The latter stimuli were perceived to be significantly smaller than four of the meaningful stimuli (the happy stick figure [item 6], the diagonal line [item 3; $M=6.95$ for both stimuli], the S shape [item 8], and the sad stick figure [item $5 ; M=6.86$ and 6.87, respectively]). The scores of the sexes did not differ, either as a main effect or in interaction with the stimuli $(F<1)$.

\section{Discussion}

The physiognomic stimulus taketa, as predicted, appeared larger than it actually was. The lines judged equal to it were actually physically larger. In contrast, size enhancement was not found for the physiognomic maluma and the two physiognomically neutral stimuli. These neutral stimuli, however, were actually smaller than both taketa and maluma (although not significantly in the latter case) and smaller than several meaningful shapes. Taketa's estimated size was also significantly larger than one of the familiar stimuli.

The reasons for taketa's enhanced size and the absence of an effect for maluma are discussed in Experiment 2. In Experiment 2, subjects judged the distances of cards on which the stimuli were mounted, rather than the stimuli themselves. Since those results, reported later, are consistent with the findings for size and the hypothesized augmenting role of physiognomy, confusion of height with area was probably not a major factor.

\section{EXPERIMENT 2}

\section{Physiognomic Distance Perception}

Given the close relationship between size and distance perception (e.g., large means close), taketa's apparent large size should affect its apparent distance. Consider the following situation: Two equal-sized cards, one blank and the other containing the physiognomic stimulus taketa, are placed equal distances from an observer. The largeappearing taketa (Experiment 1) should affect the card's apparent size in the same way that its shape influenced color perception (Lindauer, 1986). The card with taketa should therefore appear larger than it actually is. Consequently, the card with taketa should appear farther away than the blank card: Although the retinal sizes of the physiognomic and blank cards would be equal (the two cards are, after all, at the same distance and the same size), only a large object located at a greater distance could have a retinal image equal to that of a close object. Thus, subjects instructed to set the two stimuli at the same distance should place the larger-appearing taketa card farther away from the blank card (i.e., taketa's distance should be increased to make it appear equivalent in distance to the blank card). Thus, taketa will seem to be at the same distance as a neutral stimulus when it is actually farther away. These effects would not apply to the physiognomic maluma or to the two neutral stimuli, whose sizes were not augmented in Experiment 1.

\section{Method}

Twenty-one subjects ( 7 men and 14 women), freshmen volunteers for credit from several undergraduate introductory psychology classes, participated in individual sessions. All had normal (self-reported) or corrected-to-normal vision.

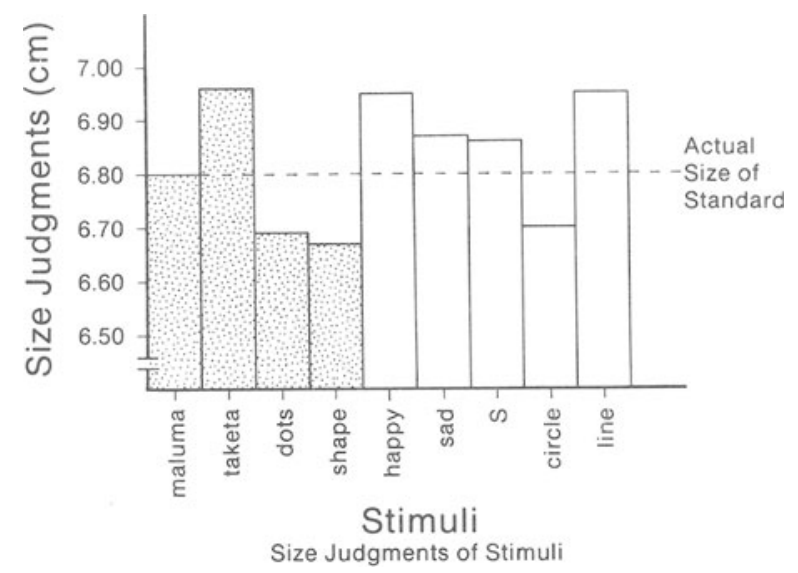

Figure 2. Size judgments in Experiment 1. The four unfamiliar stimuli are distinguished by darkened areas. 
The four critical and five filler stimuli used above and the previously omitted wavy line (item 4) were reproduced (Figure 1). They were enlarged into black-outline figures set against a white background, and then mounted on white, equal-sized $(10 \times 7 \mathrm{~cm})$ cards. Although some stimuli unavoidably covered more area on the cards, the subjects were reminded that it was the card and not the shape whose distance was to be matched. ${ }^{2}$

The subjects were instructed "to move the card until [they believed] it to be equal in distance to the blank card," that is, to set the standard and comparison stimuli so that "both are the same distance away from you." They were also told that the starting points would vary in direction and distance on each trial, and that they would "always have to move the card, at least a little." The blank standard card was always stationary. A lighted and otherwise empty enclosure provided a nearly cueless environment. ${ }^{3}$ The subjects viewed the two cards simultaneously through a $12.5 \times 6.25 \mathrm{~cm}$ aperture in the viewing box.

The subject was seated $3 \mathrm{~m}$ from the aperture of the viewing enclosure (and $3 \mathrm{~m} 15 \mathrm{~cm}$ from the standard; visual angle $=0.19^{\circ}$ ). The subject's head was held stationary on a chinrest that was adjusted to position his/her eyes on a straight line with the stimulus cards. The subjects received six trials for each stimulus: two were monocular and one was binocular (a hand-held mask covered one eye in the monocular condition); in each eye condition, the stimulus was shown in ascending or descending order (i.e., the stimulus started in a position far from or close to the subject, respectively). The starting direction of each trial, either ascending or descending, and the first eye covered in the monocular trial were alternated. The distance of the comparison stimulus from the standard at the start and the order of the stimuli shown were randomly determined (by a table of random numbers).

The comparison stimulus could be moved only in a regular progression (i.e., incrementally toward or away from the subject) rather than in a trial-and-error fashion. When the distance-matching task was completed, the amount of error was (silently) read directly from the calibrated scale located on the top of the viewing box. Overestimations (recorded as " +"' in the Results section) refer to settings of the comparison stimulus that are located away from the subject and the standard; underestimations (indicated as "-" in the Results) are settings of the comparisor stimulus that lie between the subject and the standard. If the standard and comparison stimuli were set equally, the distance was recorded as " 0 ."

Prior to the start of the test trials, the subjects received six practice trials with a sample stimulus. The comparison stimuli were removed from the viewing area and replaced without being seen by the subject until each trial was ready to begin.

Each subject received six trials with each of the 10 stimuli (for a total of 60 trials): two were monocular (one with each age) and one was binocular, and each condition was presented for one ascending and one descending trial. Negative values (from underestimated judgments) were eliminated from the analysis by adding five to all scores (and removed in the Results section).

\section{Results}

The distance settings for the stimuli significantly differed from one another $[F(9,171)=2.16, p<.05]$. However, the distance judgment significantly interacted with the type of trial, depending on whether it was ascending or descending $[F(9,171)=2.79, p<.01$; Figure 3]; consequently, each type of trial was examined separately. (Scheffé tests were used throughout.)

Consider the descending trials first (indicated as "Start Near' on Figure 3). The card mounted with taketa was set farther away from the standard (and the subject) than any other stimulus $(M=1.05 \mathrm{~cm})$ : taketa was reported to be equal to the standard card when it was actually positioned behind the standard card. Taketa's distance significantly differed $(p<.05)$ from the distances of the two neutral stimuli, the unfamiliar dots and the shape (items 9 and 10a, respectively); it did not differ, however, from that of the physiognomic maluma (item 1). Taketa also differed in distance from the meaningful circle and " $S$ " figures (items 7 and 8, respectively). (The circle was also set significantly closer than the wavy line and the happy stick figure, items 4 and 6 , respectively.)

On the ascending trials, the stimuli were initially placed far away from the observers and then brought closer (indicated as "Start Far" on Figure 3). The ascending trials, in general, resulted in significantly more errors (i.e., underestimated distances) than the descending trials $[M \mathrm{~s}=$ 1.44 and 0.40 , respectively; $F(1,19)=26.80, p<.01$ ] In other words, the stimuli generally moved past the standard and were placed closer to the subjects.

Taketa's distance setting, like the other stimuli, was also underestimated $(M=-1.11)$. However, in comparison with all the other stimuli but one (the filler circle, item 7), taketa resulted in the least error: It was stopped farther away from the subjects than the other stimuli; because it was stopped sooner, there was less underestimation of taketa's distance. This difference was significant $(p<.05)$, however, only in relation to the wavy line filler stimulus (item 4). (The circle, the only other stimulus that was significantly affected on the descending trials, also differed from the wavy line, as well as from the nonsense shape, item 10a, and the sad stick figure, item 5.)

The distance settings for taketa were not affected by either the sex of the subjects or the viewing condition (monocular/binocular) under which observations took place. Furthermore, neither the sex nor the viewing condition affected the distance settings of the stimuli; that is, neither the interaction of eye condition and stimuli $[F(18,342)=1.06, p>.05]$ nor that of sex and stimuli $(F<1)$ was significant.

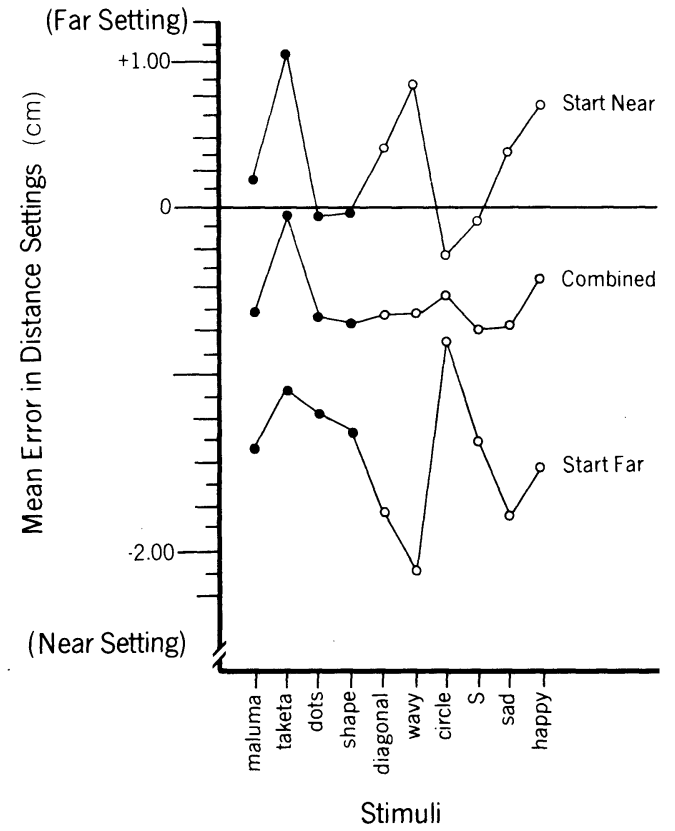

Figure 3. Distance judgments in Experiment 2. The four unfamiliar stimuli are distinguished by their darkened circles. 


\section{Discussion}

The card containing the physiognomic shape taketa was, as expected, placed at a greater distance from a neutral blank card than were cards that contained the two neutral stimuli. Taketa was placed even farther away than a few of the meaningful filler stimuli. The apparent distance of the card with taketa was increased because the shape's apparent size was enhanced (Experiment 1): Because taketa appeared larger than it actually was, viewers compensated by setting its card at a distance that decreased its retinal angle. Its apparently large size was thereby reduced so that its distance appeared equal to the standard neutral stimulus. Thus, the physiognomy of taketa affected distance just as it affected colors (Lindauer, 1986).

The distance effect was illustrated in different ways on both types of trials (in which starting points differed). On the descending trials, taketa was reported as looking equal in size to the standard when it was actually placed far away from the standard (and the subjects). On the ascending trials, taketa was stopped farthest from the subjects. As taketa moved toward the viewer, it did not have to be set as close as the other stimuli: it already looked big enough.

Taketa's distance did not differ from most of the recognizable filler shapes. The familiar stimuli had several advantages over taketa: they were imbued with more associations (and memories of known sizes), and several had affective connotations as well (e.g., the happy and sad stick figures). These characteristics might have contributed to their distance (and size) judgments. For example, subjects might have referred them to a common scale. Nevertheless, estimates of taketa's distance (and size) were equal to nearly all of the meaningful stimuli, even though taketa was meaningless. In contrast, the distance estimates for the two unfamiliar and neutral stimuli (the dots and shape) were not equivalent to those for the meaningful shapes; unlike taketa, they were not imbued with physiognomy.

The distance settings for maluma, the "quieter" physiognomic stimulus, fell between those of taketa and the nonphysiognomic neutral stimuli (Figure 3). Although maluma's distance was not significantly affected, because its size was not augmented (Experiment 1), its physiognomy nevertheless made it different from the two neutral stimuli. Hence, although the neutral stimuli's distance settings differed from taketa on the descending trials, maluma did not. In color perception, as well as in imagery and preference ratings (Lindauer, 1986), taketa dominated the findings more than maluma did, but maluma was distinguished from the neutral stimuli.

\section{GENERAL DISCUSSION}

The tasks emphasized a perceptual response: The standard and comparison stimuli were in full view of the subjects, so that judgments were concrete and direct rather than based on guessing or memory. An unfamiliar and meaningless shape such as taketa is unlikely to remind people, at least in consistent ways, of any particular, obvious, or common association (i.e., as something big and distant). Are objects with jagged angles usually considered large? Far away? Subjects in the size-matching task of Experiment 1 reported that they felt rather hopeless about their ability to respond accurately. Subjects did not report, at least spontaneously, that they were estimating, calculating, inferring, or taking things into account. When asked, subjects were unable to indicate how they made their judgments. If any mediating processes were involved (to the extent they can be consciously expressed), they were minimal or irrelevant. Taketa's size and distance differences, therefore, were not simply imagined, remembered, labeled, or calculated: Taketa looked different in size and distance.

This perceptual analysis is congruent with those of researchers who argue that physiognomy is a "given," that is, a nonmediated and autonomous quality that is embedded in input or at least occurring close to reception. According to Werner (1956; Werner \& Wapner, 1952), physiognomy demonstrates the holistic (undifferentiated) character of perception. According to Gestalt psychologists (Arnheim, 1972), physiognomy is an emergent phenomenon, revealing the underlying isomorphism (physiological parallel) between cognition and affect. According to Gibson (1979), an object's physiognomy tells perceivers about itself (i.e., its affordance).
Thus, affect and perception, at least in the case of physiognomy, supplement one another. Whether the two occur simultaneously, at the onset of stimulation (e.g., Gibson, 1979), cannot be answered on the basis of the present data. The results do suggest, however, that physiognomic perception took place spontaneously and relatively quickly.

How were taketa's size and distance (and color; Lindauer, 1986) affected? An analysis of taketa's phenomenological properties (Lindauer, 1984a), along with some principles from Gestalt psychology (Arnheim, 1972), suggest some possibilities. Taketa can be described as a bold, aggressive, and thrusting object. These qualities contrast with the other stimuli whose sizes and distance judgments were undistinguished. Maluma is a subdued, peaceful, and nonassertive shape. The two other unfamiliar shapes (the relatively meaningless array of dots, item 9, and the amoeba-like shape, item 10a) are quite nondescript. A "sharpening" process could have been prompted by taketa's overall angular shape, as well as by its individual sections (perhaps because of their irregular orientations). Other "field forces," such as good continuation, would have exaggerated taketa's features even more. These "demands" prevented closure (a "closing" or "tightening up" of its areas) and instead encouraged an "opening up." These stimulus characteristics, and the tensions they created, hint at how taketa's size and distance (and color) may have been enhanced, as well as the ease with which this shape can be labeled as aggressive and assertive.

\section{REFERENCES}

ARnheim, R. (1972). The Gestalt theory of expression. In R. Arnheim (Ed.), Toward a psychology of art: Collected essays (pp. 51-73). Berkeley, CA: University of California Press.

Comalli, E. E., JR. (1960). Studies in physiognomic perception: VI. Differential effects of directional dynamics of pictorial objects on real and apparent motion in artists and chemists. Journal of Psychology, 49, 99-109.

GiBson, J. J. (1979). The ecological approach to visual perception. Boston: Houghton Mifflin.

KOHLER, W. (1947). Gestalt psychology. New York: Liveright. (Original work published 1938)

LeiJonhielm, C. (1967). Colours, forms and art. Stockholm: Almquist \& Wiksell.

LiNDAUER, M. S. (1984a). The phenomenological method in psychology. In R. J. Corsini (Ed.), Wiley encyclopedia of psychology: Vol. 3 (pp. 42-43). New York: Wiley.

LiNDAUER, M. S. (1984b). Physiognomic perception. In R. J. Corsini (Ed.), Wiley encyclopedia of psychology: Vol. 3 (pp. 34-35). New York: Wiley.

LiNDAUER, M. S. (1986). Perceiving, imaging, and preferring physiognomic stimuli. American Journal of Psychology, 99, 233-255.

LYMAN, B. (1979). Representation of complex emotional and abstract meanings by simple forms. Perceptual \& Motor Skills, 49, 839-842.

SCHLEsinger, L. B. (1980). Physiognomic perception: Empirical and theoretical perspectives. Genetic Psychology Monographs, 101, 71-97.

WERNER, H. (1956). On physiognomic perception. In G. Kepes (Ed.), The new landscape in art and science (pp. 280-282). Chicago: Theobald.

WERNER, H., \& WAPNER, S. (1952). Toward a general theory of perception. Psychological Review, 59, 324-338.

\section{NOTES}

1. The wavy line (item 4) was not included. Its small vertical height did not permit a sufficient number of comparison stimuli to be created.

2. For example, the nonsense shape (item 10a), the nonsense dots (item 9), and maluma (item 1) covered maximum areas of $2 \times 2.5 \mathrm{~cm}$, $4.5 \times 2.5 \mathrm{~cm}$, and $5.5 \times 5.5 \mathrm{~cm}$, respectively.

3. Shadows, texture, and some perspective cues could not be completely eliminated.

(Manuscript received for publication August 15, 1987.) 